\title{
Combinatorial Design to Decipher Novel Lead Molecule against Mycobacterium tuberculosis
}

\author{
Rehman Ajijur ${ }^{1 \mathbb{D}}$, Akhtar Salman ${ }^{2 \mathbb{D}}$, Khan Mohammad Kalim Ahmad ${ }^{3, * \mathbb{C}}$ \\ 1 Department of Biotechnology, RR Institute of Modern Technology, Lucknow-226026, Uttar Pradesh, India; \\ rehman.ajijur@gmail.com (R.A.); \\ 2 Department of Bioengineering, Integral University, Lucknow-226026, Uttar Pradesh, India; sakhtar@iul.ac.in (A.S.); \\ 3 Department of Bioengineering, Integral University, Lucknow-226026, Uttar Pradesh, India; mkakhan@iul.ac.in \\ (K.M.K.A.); \\ * Correspondence: mkakhan@iul.ac.in;
}

Scopus Author ID 57193235237

Received: 3.01.2021; Revised: 30.01.2021; Accepted: 31.01.2021; Published: 7.02.2021

\begin{abstract}
Aspartate-semialdehyde dehydrogenase (ASADH) of DAP/lysine pathway plays a crucial role in sustainable growth and pathogenicity of Mycobacterium tuberculosis (Mtb) via reductive dephosphorylation of the $\beta$-aspartyl phosphate (AP). Inhibition of ASADH through different lead molecules has been gaining high impetus due to its indispensable role in the pathogen's survival. In the present study, we aimed to decipher the novel lead molecule against $M t b$. The AP, a substrate of the DAP/lysine pathway, was used as a template to design new lead molecules to advance the understanding of the molecular inhibition mechanism of $M t b$-ASADH. Monodentate and bidentate groups at three different substitution sites of AP were considered to generate a virtual library of new molecules using the combinatorial approach of the LeadGrow module of the VLifeMDS package. These substrate analogs were sifted through ADRXWS drug-likeness descriptors of the module above. Multi-scoring docking was achieved using Biopredicta, Molecular Virtual Docker, and AutoDock Tools. The adopted combinatorial approach yielded 6000 new molecules that reduced to 4979 plausible hits after lead-like filtration. The post-analysis of ADMET and molecular docking exhibited two pro-lead molecules, namely AP0600 and AP0639. The study delineates the substantial understanding of the Mtb-ASADH inhibition mechanism that would undoubtedly accelerate the pace of antitubercular design, thereby gaining more in-depth knowledge to eradicate tuberculosis across the globe.
\end{abstract}

Keywords: combinatorial design; DAP/lysine pathway; $\beta$-aspartyl phosphate; ASADH; molecular docking.

(C) 2021 by the authors. This article is an open-access article distributed under the terms and conditions of the Creative Commons Attribution (CC BY) license (https://creativecommons.org/licenses/by/4.0/).

\section{Introduction}

Tuberculosis (TB), one of the most infectious maladies caused by Mycobacterium tuberculosis (Mtb), is considered a vicious destroyer of public health worldwide. According to the Global Tuberculosis Report 2019, 10 million people got infected, in which 1.2 million deaths among HIV-negative and 251000 deaths among HIV-positive patients were observed in 2018. The diminution of $27 \%$ in HIV-negative and $60 \%$ in HIV-infected TB population compared to incidents that happened in 2000 reflects a progressive sign towards the attainment of Sustainable Development Goals (SDGs) 3 by 2030 [1]. In the current digital technology era, identifying causes impeding any successful attainment goal is not a terra incognita to the trailblazers. The flagship initiative "Find. Treat. All. \#EndTB" of three world leaders, including WHO, the Stop TB Partnership, and the Global Fund committed to defeating epidemics will 
be a milestone towards providing effective diagnosis, treatment, and care to the patients. Despite this cumulative effort, the condition in India, Nigeria, Indonesia, and the Philippines, where a robust technological breakthrough is required to improve detection of TB incidents, their diagnosis, and treatment [1]. The sputum smear microscopy, rapid molecular tests, culture-based methods, and sequencing technologies are well known TB diagnostic tests [2-5]. However, in the past few years, no new robust method and technique were seen on development that produces accurate results. Albeit, more than $85 \%$ of drug-susceptible TB disease is successfully detected and treated through a 6-month regimen of isoniazid, rifampicin, ethambutol, and pyrazinamide [6]. Moreover, the Bacillus Calmette-Guerin (BCG) is a bit primitive but effective and widely used to prevent childhood TB-meningitis and miliary disease [7].

With the advent of high-throughput technology, frequently new drug molecules are being designed and developed. Presently, 23 drug candidates are on the clinical trials' positive note suitable to recuperate different forms of TB infections, viz. Drug-susceptible, multidrugresistant (MDR), and latent TB. Additionally, 14 vaccines, respectively 3, 8, \& 3, are under different clinical trials phases [1]. Likewise, another experimental vaccine, M72/AS01E, is being developed by GlaxoSmithKline (GSK) and AERAS, which outcomes of phase II are quite promising $[1,8]$.

Notwithstanding the availability of significant measures, MDR, and extensively drugresistant (XDR), TB seems one of the most difficult conundrums for the SDGs and the End TB Strategy $[1,9]$. Therefore, identifying new chemical entities and bioisosteres of relatively low molecular weight is of more significant concern to the pharmacopeia and traditional combinatorial design. It is either achieved by modifying the existing drugs through combinatorial approaches or manipulating inhibitors of well-known molecular targets to enhance efficacy, selectivity, and pharmacokinetic properties. Once the compound is identified, it undergoes ADMET filtration to ensure whether it has toxic and mutagenic properties or not; if the compound has negligible toxicity and mutagenicity, either is considered as a potential lead molecule.

The metabolic pathways of all living organisms in which numerous bio-catalytic reactions are occurring to produce molecules that are essentially needed to sustain their life. Due to significant differences, most microorganisms synthesize the required metabolic building blocks and nutrients, while mammals obtain most of these from different nutritive sources. These substantial differences provide various enzymes, which laid the foundation for promising drug targets [10]. Aspartate-semialdehyde dehydrogenase (ASADH) is an enzyme facilitating the biosynthesis of critical metabolites of the DAP/lysine pathway, viz. dipicolinate, UDP-MurNAc-pentapeptide, and an essential amino acid lysine [11, 12]. Former both metabolites are respectively essential for sporulation and peptidoglycan cross-linking via covalent interaction with $\mathrm{D}$-alanyl moieties of vicinal chain to generate murein polymers providing stability and rigidity to the pathogen cell wall [10,13-15]. Moreover, It is also well established experimentally that de novo biosynthesis of lysine is of the utmost importance to the survival of $M t b$ during infection, even though adequacy in the host. Therefore, inhibition of the DAP/lysine pathway through the ASADH enzyme is a promising drug target against $M t b[14-16]$.

In the proposed work, in silico approach was adopted to identify novel leads against $M t b$-ASADH through combinatorial design. The $\beta$-aspartyl phosphate (AP), a substrate of the DAP/lysine pathway, was selected as a template molecule to generate a virtual library of 
AP-analogues through the LeadGrow module of the VLifeMDS package [17]. The top-ranked hits of new chemical entities were subjected to drug-likeness and ADMET filtration followed by comprehensive multi-scoring molecular interactions using three different computational tools, namely Biopredicta, Molecular Virtual Docker (MVD), and AutoDock Tools [18-20] to get consistent pro-lead molecules. Subsequently, the top 2 hits (AP0600and AP0639) along with AP and two known inhibitors (ZINC04203124, NSC51108) [21, 22] were simulated computationally. The post-docking simulation depicted AP0639 showing strong and stable molecular interactions to the target protein's critical residues. Moreover, the lead molecule reported here may help design a novel AP-antagonist, thereby improving target inhibition competence.

\section{Materials and Methods}

\section{1. $3 D$ structure retrieval of $M t b-A S A D H$.}

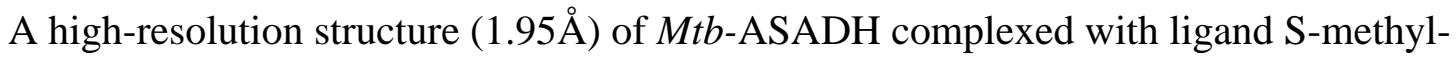
L-cysteine sulfoxide (CID: 182092) and sulfate ion was extracted from RCSB PDB (https://www.rcsb.org/structure/3TZ6). The only apoprotein of the complex was carried forward to prepare 3D-files suitable for docking simulation by removing undesired ions and molecules. The coordinates of co-crystallized inhibitors were considered for molecular interactions of ligands [23]. The CHARMm force field was assigned to optimize the apoprotein, followed by energy minimization using steepest descent and conjugate gradient algorithm to remove the steric hindrances [24, 25].

\subsection{Combinatorial design, drug-likeness, and ADMET prediction.}

All possible permutations and combinations of different monodentate and bidentate functional moieties of the LeadGrow module at three substitutional sites of AP were checked [17], and a combinatorial library of 6000 AP-analogues created. These nascent ligands were tested on 'ADRXWS' drug-like descriptors, which respectively stands for H-bond acceptors $(<=10)$, H-bond donors $(<=5)$, rotatable bond count $((<=10)$, lipophilicity (X $\log \mathrm{P}<=5)$, molecular weight $\left(<=500\right.$ Dalton), and polar surface area $\left(<=60 \AA^{2}\right)$ [26-28]. Further, the ADMET (absorption, distribution, metabolism, elimination, and toxicity) assessment of ADRXWS-satisfied compounds is accomplished through PreADMET online tool that predicts the pharmacological and toxicological properties [29-37]. The chemical structure of AP and its top 8 analogs are shown in Figure 1.

\subsection{Docking simulations.}

BioPredicta tool of VlifeMDS (https://www.vlifesciences.com/) [38, 39] MVD (http://www.clcbio.com) [40] and AutoDock Tools 4.0 (http://autodock.scripps.edu/) [20] were used for molecular interaction studies of ligands and protein.

\subsubsection{BioPredicta.}

It employs a cascaded approach of genetic algorithm and piecewise linear pairwise potential (PLP). The Merck molecular force field (MMFF) was used to compute the ligand's best pose into the active site of protein molecule with the least docking score, including various energy terms viz. hydrogen bonding der Waals interactions, hydrophobic effects, and 
deformation penalty [41]. The other parameters were kept as default to execute molecular docking.

\subsubsection{MVD.}

It is based on the MolDock scoring function that employs heuristic search techniques to find out the most stable and energetically minimized ligand conformation. The MolDock scoring function is a sort of PLP dealing with the electrostatic potential and H-bonding as energy attributes. Further, a MolDock-rerank score was applied to hone molecular interactions' accuracy $[42,43]$.

\subsubsection{AutoDock tools.}

Docking simulation of virtually designed ligands with $M t b$-ASADH was performed using AutoDock Tools 4.0 to obtain the most promising binding poses among them. The pdbqt files for protein and ligands, grid parameter file (gpf), and a docking parameter file were generated to run the program. A grid-box with enough space was created wherein ligand can move amenably. The grid points of $60 \AA$ in each $\mathrm{x}, \mathrm{y}$, and $\mathrm{z}$ axes were drawn, having a distance of $0.357 \AA$ between two consecutive grids. The Lamarckian Genetic Algorithm (LGA) performs better than the genetic algorithm alone. Simulated annealing was set to rigid-protein docking. Ten search attempts were made for ligand molecules. 250000 energy evaluations and 27000 generations before the termination of the LGA run were considered. For other constraints, software default values were taken. During the docking process, a maximum of 20 different conformations of ligand was considered. The ligand's best pose in terms of lowest free energy of binding $(\Delta \mathrm{G})$ and inhibition constant was carried forward for further interactions analysis [20, 44-47]. The flow chart of the adopted methodology is shown in Figure 2.

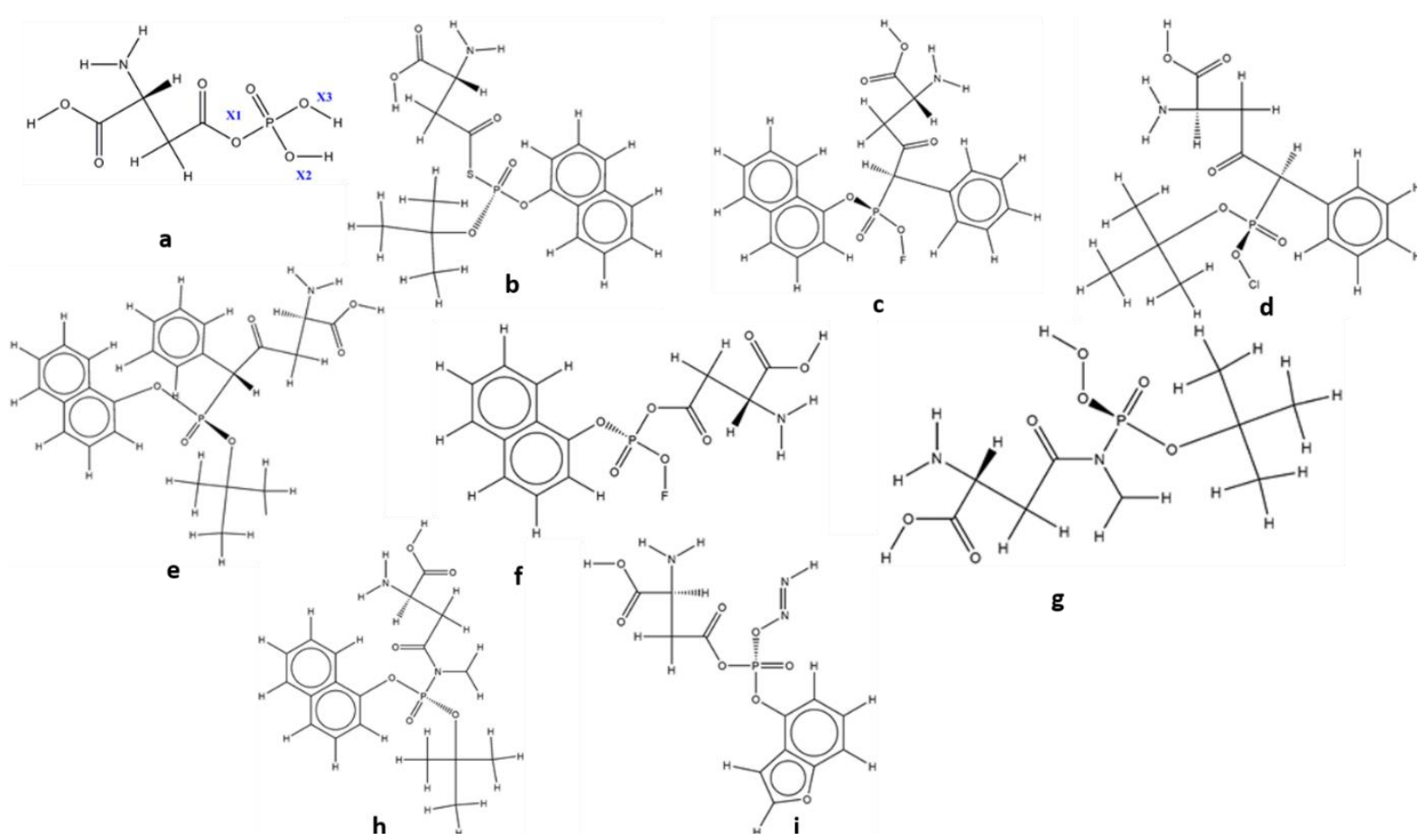

Figure 1. Chemical strucrure of ligands (a) AP (b) AP0592 (c) AP0600 (d) AP0609 (e) AP0611 (f) AP0617 (g) AP0638 (h) AP0639 (i) AP0861. 


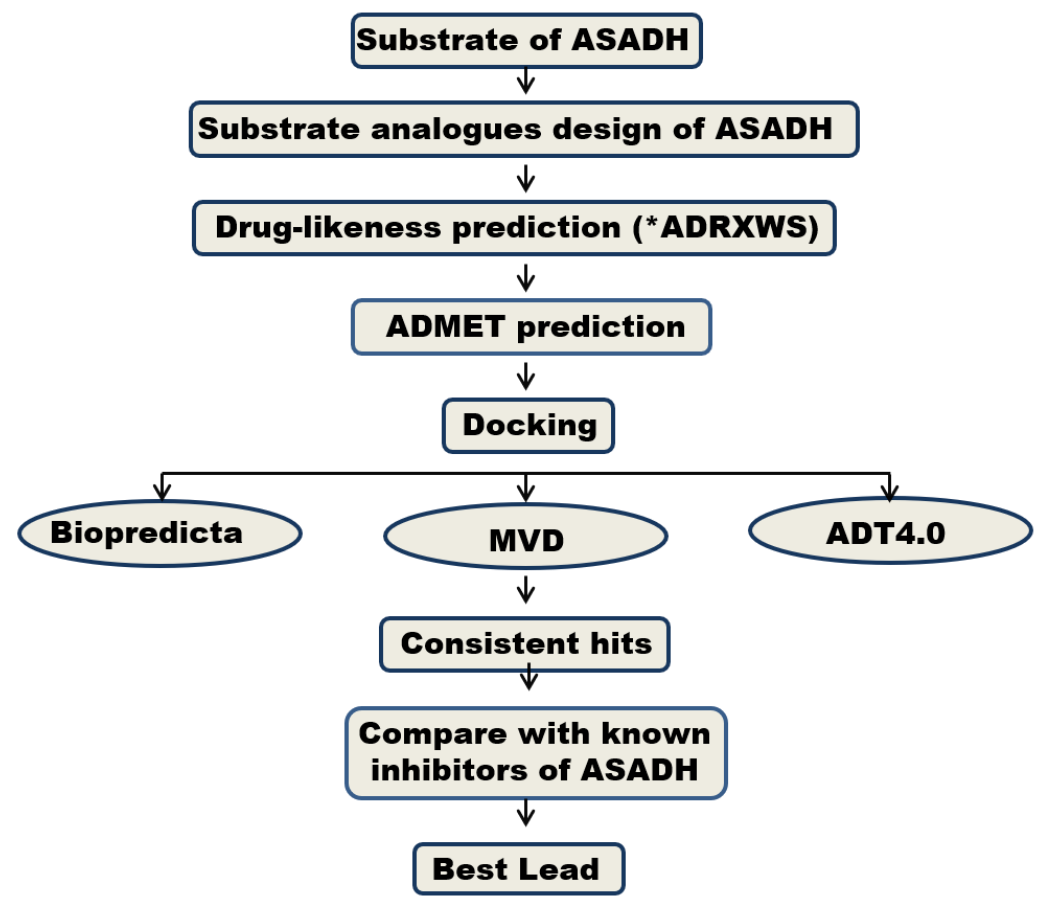

Figure 2. Flow chart of methodology. *ADRXWS respectively stands for H-bond acceptor, H-bond donor, rotatable bond count, lipophilicity, molecular weight, and polar surface area.

\section{Results and Discussion}

A combinatorial library was created to design and identify substrate analog with antitubercular potential, considering $\beta$-aspartyl phosphate (AP) as a template. Initially, AP-like 6000 compounds were designed. These molecules were subjected to RO5 filtration (4979), followed by ADMET screening (1187) before docking studies. Molecular docking studies of 1187 AP- analogs were performed for the best binding orientation prediction into the active site of $M t b$-ASADH using the same docking procedure and parameters as a template molecule. The stepwise result of the study is shown in Figure 3.

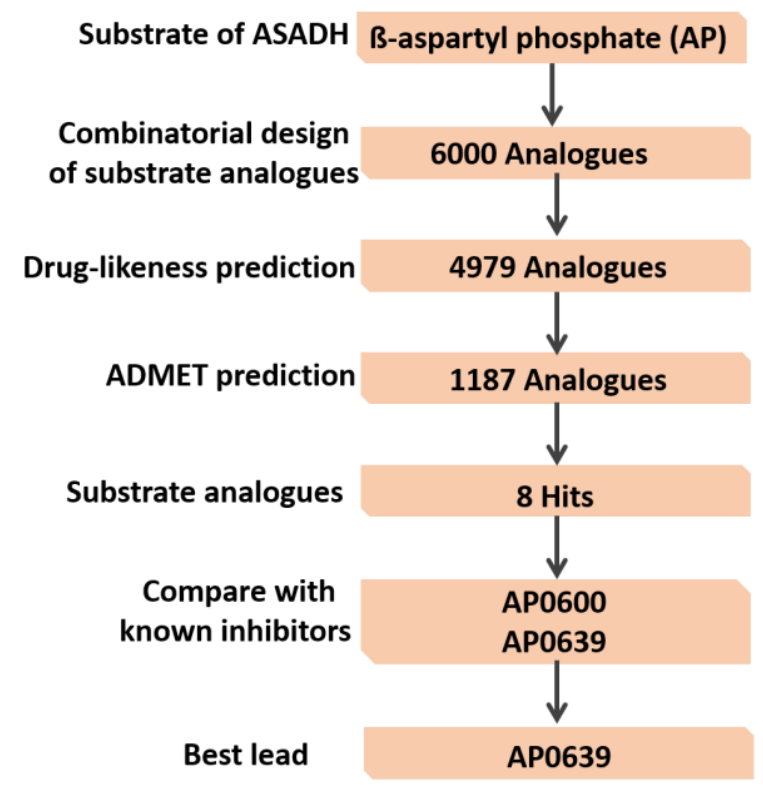

Figure 3. Flow chart of the stepwise result. AP0600 and AP0639 were compared with ZINC04203124 and NSC51108 [21, 22]. 


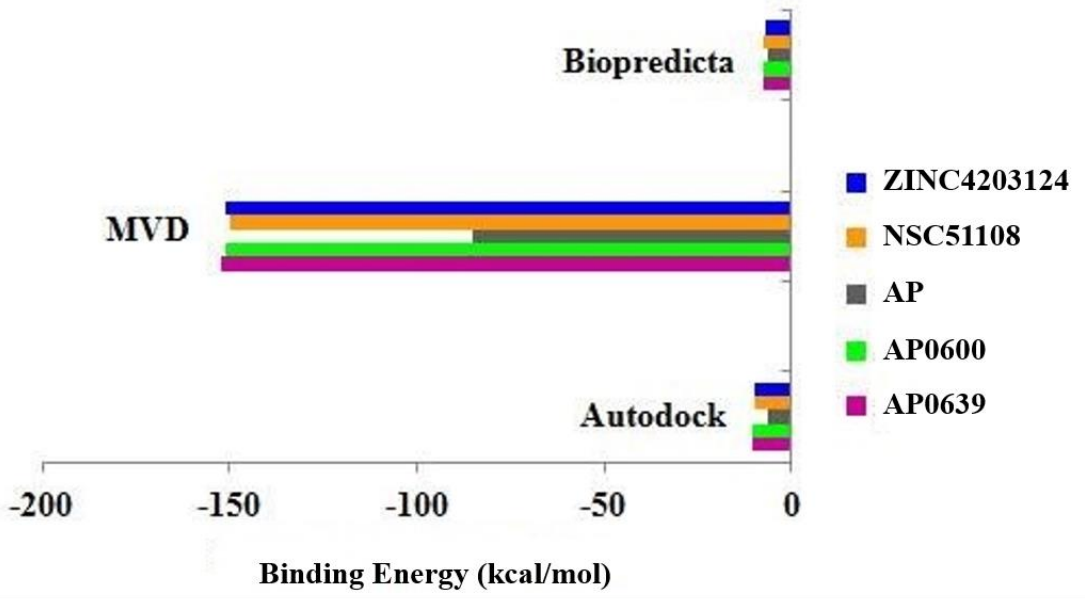

Figure 4. Docking comparison of top AP- analogs with AP and known inhibitors.

Out of 1187, only eight analogs exhibited better binding than AP and H-bond formation with the active site residue Lys171 of $M t b-A S A D H$. Furthermore, out of 8 , only two compounds (ap0861 and ap0639) were depicted as more potent inhibitors in comparison to ZINC04203124 and NSC51108 [21,22] as shown by scoring functions of adopted docking tools (Figure 4).

Binding affinity of $M t b$-ASADH with different ligands were predicted as followsAP0639 > AP0600 > NSC51108 > ZINC4203124 > AP0617 > AP0861 > AP0638 > AP0611 $>$ AP0609 > AP0592 > AP. The details of docking analysis of AP, AP-analogs, and known inhibitors are summarized in Table 1.

Table 1. Molecular interactions of top ap-analogs and their comparison with known inhibitors.

\begin{tabular}{l|l|c|c|c|c} 
S. No. & Compound ID & $\begin{array}{c}\text { Biopredicta } \\
\text { (Docking Score) }\end{array}$ & $\begin{array}{c}\text { AutoDock Tools 4.0 } \\
(\boldsymbol{\Delta G} \text { kcal/mol })\end{array}$ & $\begin{array}{c}\text { MVD 5.5 } \\
(\text { MolDock Score })\end{array}$ & No. of H-bonds \\
\hline 1. & AP0592 & -6.448392 & -7.22 & -141.725 & 1 \\
\hline 2. & AP0600 & -7.800266 & -10.73 & -156.244 & 5 \\
\hline 3. & AP0609 & -6.736073 & -7.77 & -141.983 & 2 \\
\hline 4. & AP0611 & -6.871995 & -7.82 & -142.490 & 1 \\
\hline 5. & AP0617 & -7.133096 & -9.65 & -149.056 & 3 \\
\hline 6. & AP0638 & -6.965838 & -7.95 & -143.733 & 3 \\
\hline 7. & AP0639 & -7.905518 & -10.97 & -162.140 & 4 \\
\hline 8. & AP0861 & -6.973931 & -7.98 & -143.882 & 4 \\
\hline 9. & AP & -6.199562 & -6.17 & -134.992 & 5 \\
\hline 10. & NSC51108 & -7.219866 & -9.89 & -150.917 & 5 \\
\hline 11. & ZINC4203124 & -7.197657 & -9.78 & -149.878 & 4
\end{tabular}

Moreover, GLN13, VAL14, SER71, ASN94, SER95, ARG99, ASN129, CYS130, SER160, GLY163, and LYS227 residues of Mtb-ASADH interacted with AP0639 along with four H-bond formations stabilizing the complex (Figure 5). Likewise, GLY212, GLY261, CYS130, ARG249, ASN139, LYS227, CYS247, ALA211, ASP223, and ASP225 residues of target protein played a crucial role in molecular interaction with AP0600 with five H-bonds supporting the stability of the complex (Figure 6). The known inhibitor NSC51108 interacted with ARG249, ASP223, THR131, ASN129, CYS247, LYS227, ASP225 GLN157 residues of $M t b$-ASADH along with five H-bonds (Figure 7). Similarly, other reference inhibitor ZINC4203124 engaged in molecular interaction with VAL14, GLY163, ASN94, SER95, GLY73, ARG99, LYS227, ASN129, GLY161, and GLN13 residues with four H-bonds (Figure 8). Substrate AP exhibited interaction with SER71, GLY73, ASN94, SER95, ARG99, ASN129, and LYS227 residues with five H-bonds (Figure 9). 
The collapse of antitubercular lead molecules due to MDR, XDR, and several other reasons prompts the scientific community to decipher new potential lead molecules against $M t b$ that could effectively prevent tuberculosis. Towards this direction, the combinatorial approach was utilized to predict new lead compounds. AP was chosen as a reference molecule to design 6000 new compounds using the LeadGrow module of VLifeMDS. These analogs were additionally sifted through ADRXWS descriptors that curtailed to 4979 molecules. Subsequently, ADMET forecast 1187 hits satisfying pharmacokinetic parameters that were further reduced to 8 potential hits thorough multi-scoring docking analysis through Biopredicta, MVD, and ADT. Further, the ligand AP0639 depicted more excellent stability than substrate molecules AP and known inhibitors that might be used as an inhibitor against $M t b$-ASADH.

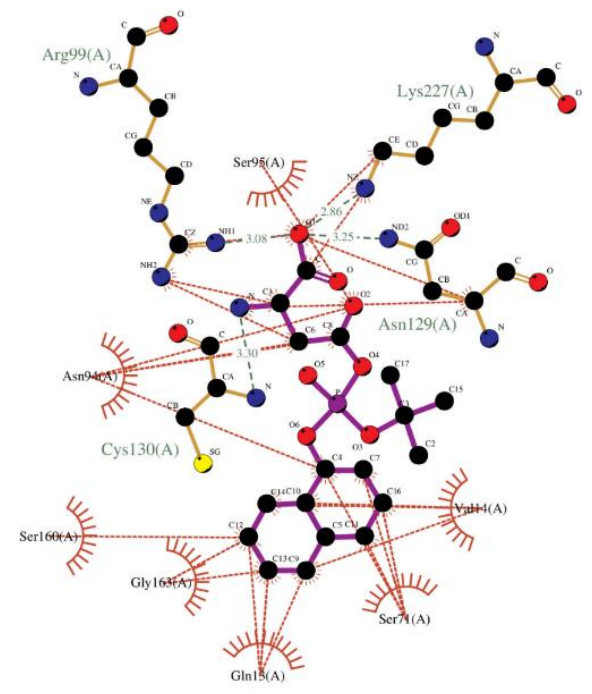

Figure 5. The Ligplot of Mtb-ASADH-AP0639 complex. Dashed lines and arcs show hydrophobic contacts and H-bonds, respectively.

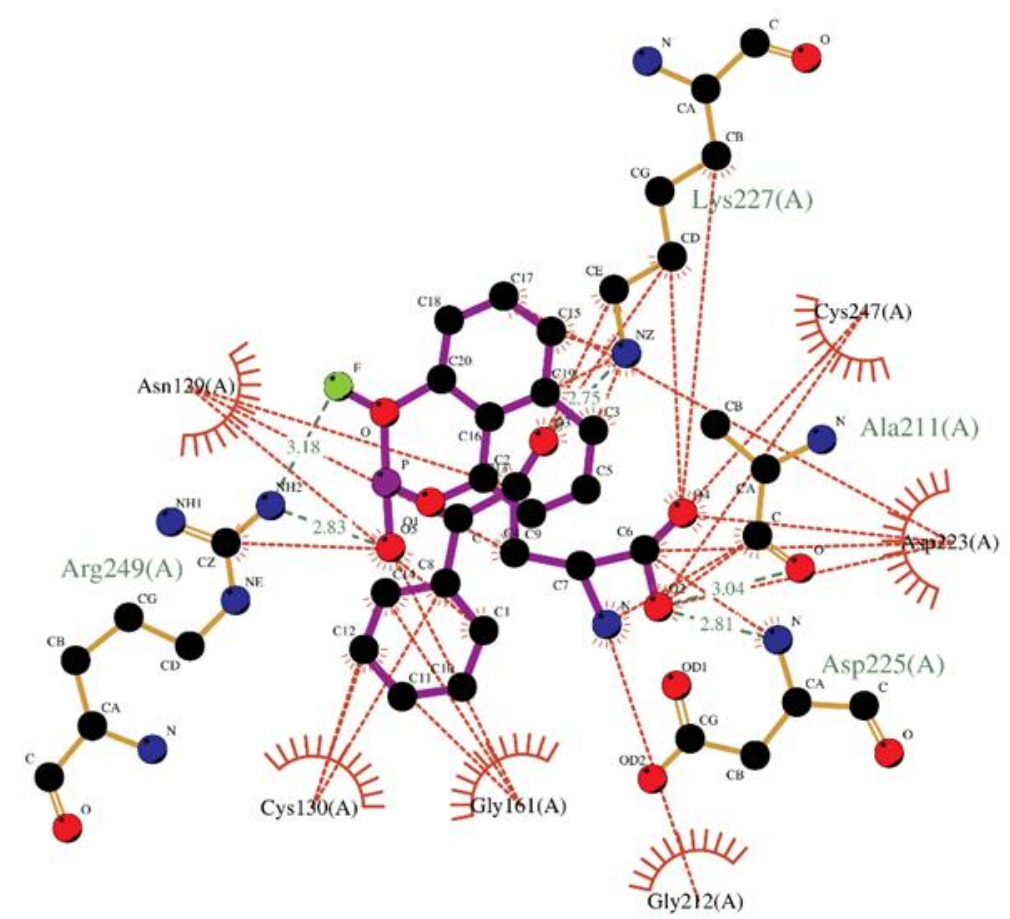

Figure 6. The Ligplot of Mtb-ASADH-AP0600 complex. Dashed lines and arcs show hydrophobic contacts and H-bonds, respectively. 


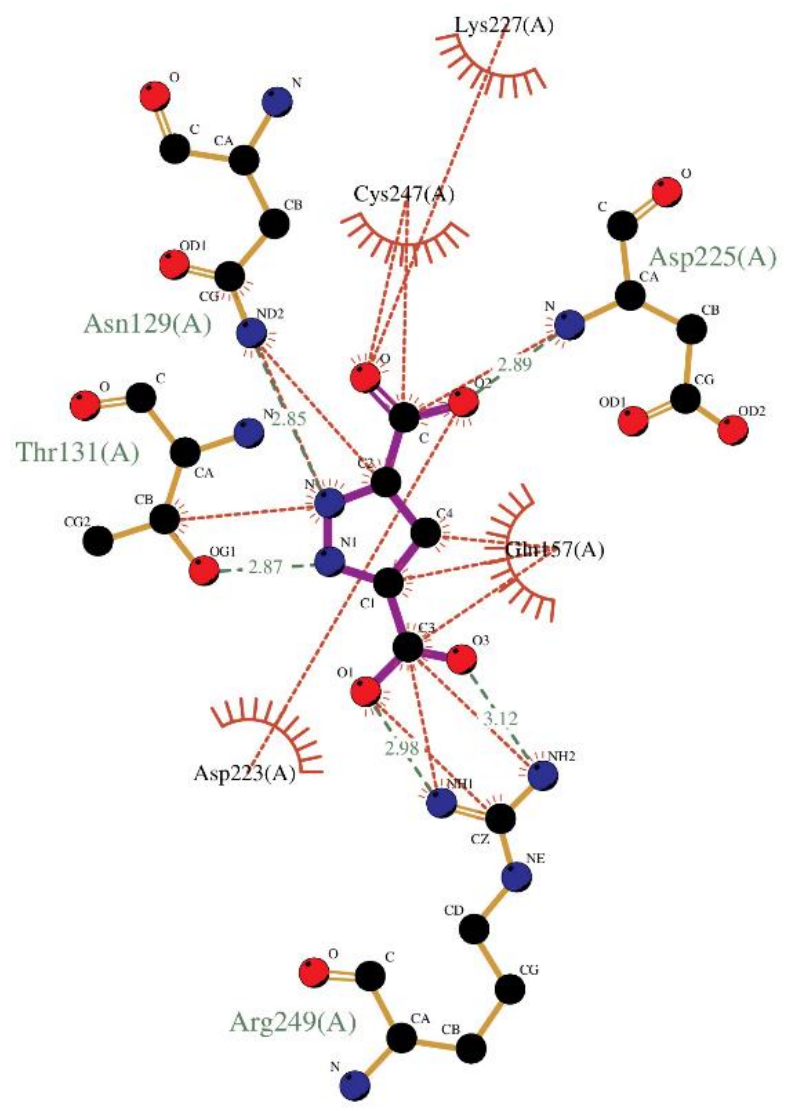

Figure 7. The Ligplot of Mtb-ASADH-NSC51108 complex. Dashed lines and arcs show hydrophobic contacts and H-bonds, respectively.

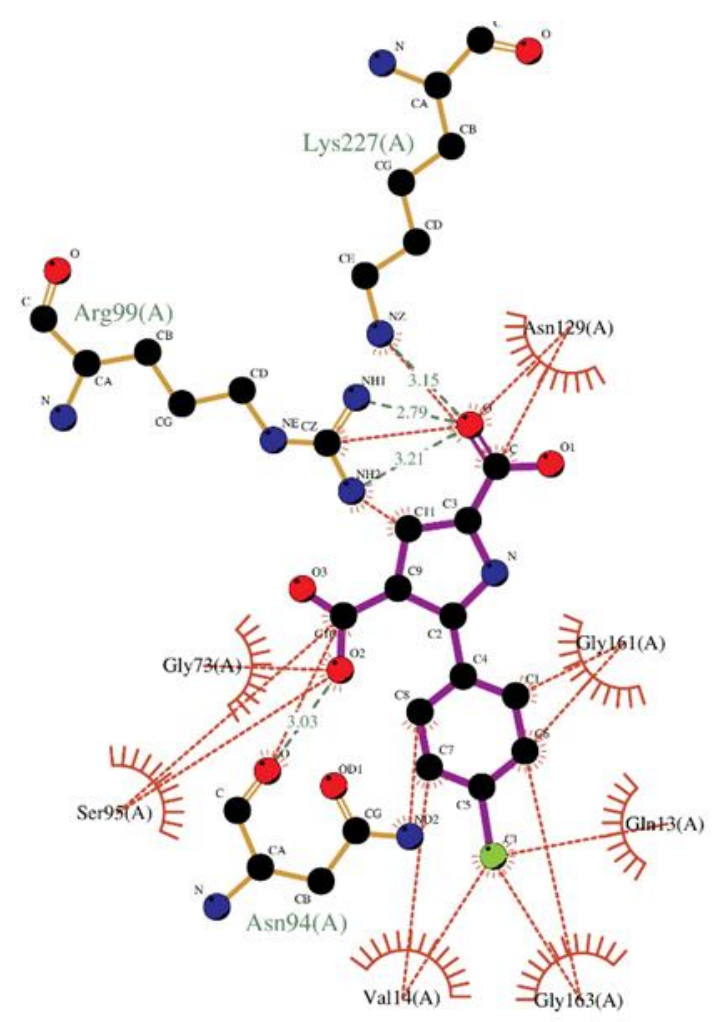

Figure 8. The Ligplot of Mtb-ASADH-ZINC4203124 complex. Dashed lines and arcs show hydrophobic contacts and H-bonds, respectively. 


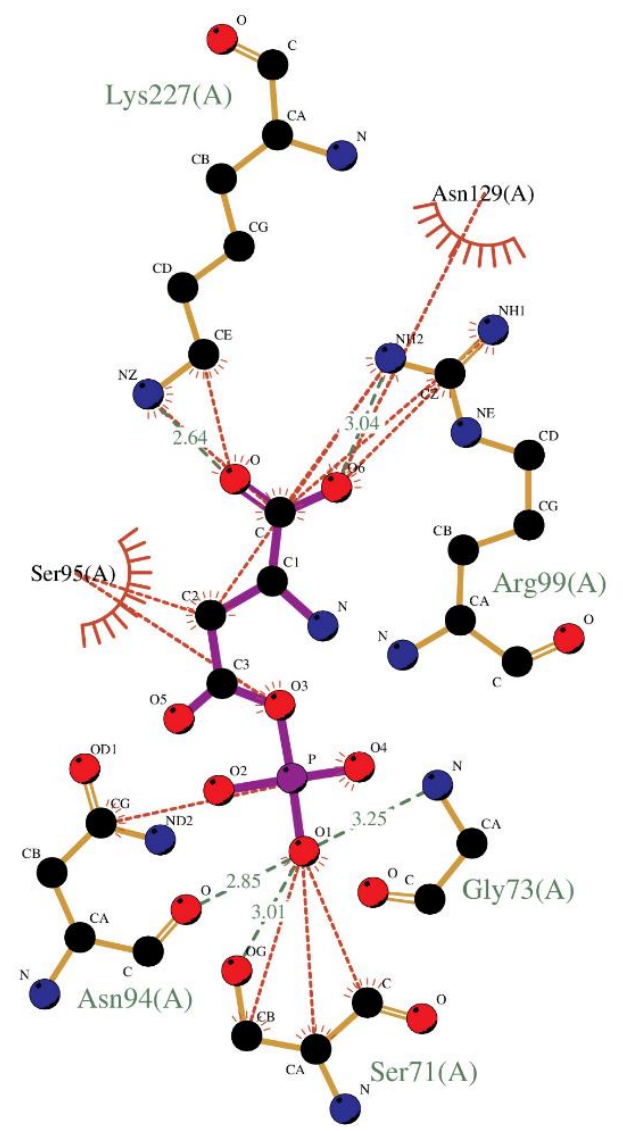

Figure 9. The Ligplot of $M t b$-ASADH-AP complex. Dashed lines and arcs show hydrophobic contacts and Hbonds, respectively.

\section{Conclusions}

Tuberculosis has been considered one of the oldest diseases for centuries and remains a significant health issue worldwide. It affects a million individuals every year and is accountable as one of the top ninth leading cause of death worldwide. It has been the primary cause of death from a single infectious disease for over five years, positioning above HIV/AIDS. It is a curable disease, but many people suffer every year. A number of them do not survive, resulting in devastating social and economic impacts. Various programs worldwide have been running to eradicate the disease by various means, whether implementing policies or designing new drugs. Adding a bit to this direction, we predicted a new lead compound AP0639 that could be considered a potential inhibitor against the DAP/Lysine pathway. The wet-lab experiments are required to validate the study's in silico findings; however, anticipated leads would undoubtedly help explore more specific and selective drug candidates against $M t b$.

\section{Funding}

This research received no external funding.

\section{Acknowledgments}

This research has no acknowledgment.

\section{Conflicts of Interest}

The authors declare no conflict of interest. 


\section{References}

1. WHO. WHO | Global Tuberculosis Report 2019. 2020.

2. Das, P.; Ganguly, S.; Mandal, B. Sputum Smear Microscopy in Tuberculosis: It Is Still Relevant in the Era of Molecular Diagnosis When Seen from the Public Health Perspective. Biomed. Biotechnol. Res. J. 2019, 3, 77-79, https://doi.org/10.4103/bbrj.bbrj_54_19.

3. Ng, K. C. S.; Supply, P.; Cobelens, F. G. J.; Gaudin, C.; Gonzalez-Martin, J.; de Jong, B. C.; Rigouts, L. How Well Do Routine Molecular Diagnostics Detect Rifampin Heteroresistance in Mycobacterium Tuberculosis? Journal of Clinical Microbiology 2019, 57, e00717-00719, https://doi.org/10.1128/JCM.00717-19.

4. Anyansi, C.; Keo, A.; Walker, B.J.; Straub, T.J.; Manson, A.L.; Earl, A.M.; Abeel, T. QuantTB - a method to classify mixed Mycobacterium tuberculosis infections within whole genome sequencing data. BMC Genomics 2020, 21, https://doi.org/10.1186/s12864-020-6486-3.

5. Carey, A.F.; Rock, J.M.; Krieger, I.V.; Chase, M.R.; Fernandez-Suarez, M.; Gagneux, S.; Sacchettini, J.C.; Ioerger, T.R.; Fortune, S.M. TnSeq of Mycobacterium tuberculosis clinical isolates reveals strain-specific antibiotic liabilities. PLoS Pathog 2018, 14, https://doi.org/10.1371/journal.ppat.1006939.

6. Yoon, S.-W.; Choi, J. C. Treatment of Pulmonary Tuberculosis. J. Korean Med. Assoc. 2019, 62, https://doi.org/10.5124/jkma.2019.62.1.25.

7. Coppola, M.; van den Eeden, S.J.F.; Robbins, N.; Wilson, L.; Franken, K.L.M.C.; Adams, L.B.; Gillis, T.P.; Ottenhoff, T.H.M.; Geluk, A. Vaccines for Leprosy and Tuberculosis: Opportunities for Shared Research, Development, and Application. Frontiers in Immunology 2018, 9, https://doi.org/10.3389/fimmu.2018.00308.

8. Tait, D.R.; Hatherill, M.; Van Der Meeren, O.; Ginsberg, A.M.; Van Brakel, E.; Salaun, B.; Scriba, T.J.; Akite, E.J.; Ayles, H.M.; Bollaerts, A.; Demoitié, M.-A.; Diacon, A.; Evans, T.G.; Gillard, P.; Hellström, E.; Innes, J.C.; Lempicki, M.; Malahleha, M.; Martinson, N.; Mesia Vela, D.; Muyoyeta, M.; Nduba, V.; Pascal, T.G.; Tameris, M.; Thienemann, F.; Wilkinson, R.J.; Roman, F. Final Analysis of a Trial of M72/AS01E Vaccine to Prevent Tuberculosis. New England Journal of Medicine 2019, 381, 2429-2439, https://doi.org/10.1056/NEJMoa1909953.

9. Coll, F.; Phelan, J.; Hill-Cawthorne, G.A.; Nair, M.B.; Mallard, K.; Ali, S.; Abdallah, A.M.; Alghamdi, S.; Alsomali, M.; Ahmed, A.O.; Portelli, S.; Oppong, Y.; Alves, A.; Bessa, T.B.; Campino, S.; Caws, M.; Chatterjee, A.; Crampin, A.C.; Dheda, K.; Furnham, N.; Glynn, J.R.; Grandjean, L.; Minh Ha, D.; Hasan, R.; Hasan, Z.; Hibberd, M.L.; Joloba, M.; Jones-López, E.C.; Matsumoto, T.; Miranda, A.; Moore, D.J.; Mocillo, N.; Panaiotov, S.; Parkhill, J.; Penha, C.; Perdigão, J.; Portugal, I.; Rchiad, Z.; Robledo, J.; Sheen, P.; Shesha, N.T.; Sirgel, F.A.; Sola, C.; Oliveira Sousa, E.; Streicher, E.M.; Helden, P.V.; Viveiros, M.; Warren, R.M.; McNerney, R.; Pain, A.; Clark, T.G. Genome-wide analysis of multi- and extensively drugresistant Mycobacterium tuberculosis. Nature Genetics 2018, 50, 307-316, https://doi.org/10.1038/s41588017-0029-0.

10. Viola, R.E.; Faehnle, C.R.; Blanco, J.; Moore, R.A.; Liu, X.; Arachea, B.T.; Pavlovsky, A.G. The Catalytic Machinery of a Key Enzyme in Amino Acid Biosynthesis. Journal of Amino Acids 2011, 2011, 1-11, https://doi.org/10.4061/2011/352538.

11. Khan, S.; Somvanshi, P.; Bhardwaj, T.; Mandal, R.K.; Dar, S.A.; Wahid, M.; Jawed, A.; Lohani, M.; Khan, M.; Areeshi, M.Y.; Haque, S. Aspartate- $\beta$-semialdeyhyde dehydrogenase as a potential therapeutic target of Mycobacterium tuberculosis H37Rv: Evidence from in silico elementary mode analysis of biological network model. Journal of cellular biochemistry 2018, 119, 2832-2842, https://doi.org/10.1002/jcb.26458.

12. Hasenoehrl, E.J.; Rae Sajorda, D.; Berney-Meyer, L.; Johnson, S.; Tufariello, J.M.; Fuhrer, T.; Cook, G.M.; Jacobs, W.R.; Berney, M. Derailing the aspartate pathway of Mycobacterium tuberculosis to eradicate persistent infection. Nature Communications 2019, 10, https://doi.org/10.1038/s41467-019-12224-3.

13. Maitra, A.; Munshi, T.; Healy, J.; Martin, L.T.; Vollmer, W.; Keep, N.H.; Bhakta, S. Cell wall peptidoglycan in Mycobacterium tuberculosis: An Achilles' heel for the TB-causing pathogen. FEMS Microbiology Reviews 2019, 43, 548-575, https://doi.org/10.1093/femsre/fuz016.

14. Jankute, M.; Cox, J.A.G.; Harrison, J.; Besra, G.S. Assembly of the Mycobacterial Cell Wall. Annual Review of Microbiology 2015, 69, 405-423, https://doi.org/10.1146/annurev-micro-091014-104121.

15. Abrahams, K.A.; Besra, G.S. Mycobacterial cell wall biosynthesis: a multifaceted antibiotic target. Parasitology 2018, 145, 116-133, https://doi.org/10.1017/S0031182016002377.

16. Peverelli, M.G.; Soares da Costa, T.P.; Kirby, N.; Perugini, M.A. Dimerization of Bacterial Diaminopimelate Decarboxylase Is Essential for Catalysis *. Journal of Biological Chemistry 2016, 291, 9785-9795, https://doi.org/10.1074/jbc.M115.696591.

17. Junaid, M.; Almuqri, E.A.; Liu, J.; Zhang, H. Analyses of the Binding between Water Soluble C60 Derivatives and Potential Drug Targets through a Molecular Docking Approach. PLOS ONE 2016, 11, https://doi.org/10.1371/journal.pone.0147761.

18. Rehman, A.; Akhtar, S.; Siddiqui, M.H.; Khan, M.K.A. In Silico Identification of Drug-Like Inhibitors against Mtb-DHDPS: A Shape-Based Approach. J. Pharm. Sci. Res. 2019, 11, 2084-2089. 
19. Bitencourt-Ferreira, G.; de Azevedo, W. F. Molegro Virtual Docker for Docking. In: Methods in Molecular Biology. 2019; pp 149-167, https://doi.org/10.1007/978-1-4939-9752-7_10.

20. Morris, G.M.; Goodsell, D.S.; Halliday, R.S.; Huey, R.; Hart, W.E.; Belew, R.K.; Olson, A.J. Automated docking using a Lamarckian genetic algorithm and an empirical binding free energy function. Journal of Computational Chemistry 1998, 19, 1639-1662, https://doi.org/10.1002/(SICI)1096987X(19981115)19:14<1639::AID-JCC10>3.0.CO;2-B.

21. Kumar, R.; Garg, P.; Bharatam, P.V. Shape-based virtual screening, docking, and molecular dynamics simulations to identify Mtb-ASADH inhibitors. Journal of Biomolecular Structure and Dynamics 2015, 33, 1082-1093, https://doi.org/10.1080/07391102.2014.929535.

22. Rajender, K.; Ratnika, S.; Purvi, S.; Ipsita, R.; Inder Pal, S.; Bharatam, P.V.; Rupinder, T.; Prabha, G. Biological Evaluation of Small Molecule Inhibitors of Mtb-ASADH Enzyme. Letters in Drug Design \& Discovery 2016, 13, 587-590, https://doi.org/10.2174/1570180813666160617102813.

23. Vyas, R.; Tewari, R.; Weiss, M.S.; Karthikeyan, S. Structures of Ternary Complexes of AspartateSemialdehyde Dehydrogenase (Rv3708c) from Mycobacterium Tuberculosis H37Rv. Acta Crystallogr. Sect. D Biol. Crystallogr. 2012, 68, 671-679, https://doi.org/10.1107/S0907444912007330.

24. Lemkul, J. A. Pairwise-Additive and Polarizable Atomistic Force Fields for Molecular Dynamics Simulations of Proteins. In: Progress in Molecular Biology and Translational Science. 2020; pp 1-71, https://doi.org/10.1016/bs.pmbts.2019.12.009.

25. Kim, S.; Lee, J.; Jo, S.; Brooks Iii, C.L.; Lee, H.S.; Im, W. CHARMM-GUI ligand reader and modeler for CHARMM force field generation of small molecules. Journal of Computational Chemistry 2017, 38, 18791886, https://doi.org/10.1002/jcc.24829.

26. Lenci, E.; Trabocchi, A. Synthetic Approaches toward Small Molecule Libraries. In: Small Molecule Drug Discovery. Elsevier, 2020; pp 1-34, https://doi.org/10.1016/B978-0-12-818349-6.00001-7.

27. Manallack, D.T.; Yuriev, E.; Chalmers, D.K. The influence and manipulation of acid/base properties in drug discovery. Drug Discovery Today: Technologies 2018, 27, 41-47, https://doi.org/10.1016/j.ddtec.2018.04.003.

28. Lipinski, C.A. Lead- and drug-like compounds: the rule-of-five revolution. Drug Discovery Today: Technologies 2004, 1, 337-341, https://doi.org/10.1016/j.ddtec.2004.11.007.

29. Tan, H.-Y.; Trier, S.; Rahbek, U.L.; Dufva, M.; Kutter, J.P.; Andresen, T.L. A multi-chamber microfluidic intestinal barrier model using Caco-2 cells for drug transport studies. PLOS ONE 2018, 13, https://doi.org/10.1371/journal.pone.0197101.

30. Zhu, J.; Yi, X.; Zhang, J.; Chen, S.; Wu, Y. Rapid Screening of Brain-Penetrable Antioxidants from Natural Products by Blood-Brain Barrier Specific Permeability Assay Combined with DPPH Recognition. J. Pharm. Biomed. Anal. 2018, 151, 42-48, https://doi.org/10.1016/j.jpba.2017.12.055.

31. Liu, W.; Jiang, X.; Zu, Y.; Yang, Y.; Liu, Y.; Sun, X.; Xu, Z.; Ding, H.; Zhao, Q. A Comprehensive Description of GluN2B-Selective N-Methyl-D-Aspartate (NMDA) Receptor Antagonists. Eur. J. Med. Chem. 2020, 200, https://doi.org/10.1016/j.ejmech.2020.112447.

32. Zhu, L.; Zhao, J.; Zhang, Y.; Zhou, W.; Yin, L.; Wang, Y.; Fan, Y.; Chen, Y.; Liu, H. ADME Properties Evaluation in Drug Discovery: In Silico Prediction of Blood-Brain Partitioning. Mol. Divers. 2018, 22, 979990, https://doi.org/10.1007/s11030-018-9866-8.

33. Bergmann, S.; Lawler, S.E.; Qu, Y.; Fadzen, C.M.; Wolfe, J.M.; Regan, M.S.; Pentelute, B.L.; Agar, N.Y.R.; Cho, C.-F. Blood-brain-barrier organoids for investigating the permeability of CNS therapeutics. Nature Protocols 2018, 13, 2827-2843, https://doi.org/10.1038/s41596-018-0066-x.

34. Jijie, R.; Barras, A.; Boukherroub, R.; Szunerits, S. Nanomaterials for transdermal drug delivery: beyond the state of the art of liposomal structures. Journal of Materials Chemistry B 2017, 5, 8653-8675, https://doi.org/10.1039/C7TB02529G.

35. Hatton, G.B.; Madla, C.M.; Rabbie, S.C.; Basit, A.W. Gut reaction: impact of systemic diseases on gastrointestinal physiology and drug absorption. Drug Discovery Today 2019, 24, 417-427, https://doi.org/10.1016/j.drudis.2018.11.009.

36. Volpe, D.A. Advances in cell-based permeability assays to screen drugs for intestinal absorption. Expert Opinion on Drug Discovery 2020, 15, 539-549, https://doi.org/10.1080/17460441.2020.1735347.

37. Pan, F.; Han, L.; Zhang, Y.; Yu, Y.; Liu, J. Optimization of Caco-2 and HT29 co-culture in vitro cell models for permeability studies. International Journal of Food Sciences and Nutrition 2015, 66, 680-685, https://doi.org/10.3109/09637486.2015.1077792.

38. Bhosale, M.; Yadav, A.; Magdum, C.; Mohite, S. Molecular Docking Studies, Synthesis, Toxicological Evaluation using Brine Shrimp (Artemia salina L.) Model and Anti-inflammatory Activity of Some N(substituted)-5-phenyl-1,3,4-thiadiazol-2-amine Derivatives. International Journal of Scientific Research in Science and Technology 2020, 151-62, https://doi.org/10.32628/IJSRST207515.

39. Das, R.; Mehta, D.K. Evaluation and Docking Study of Pyrazine Containing 1, 3, 4-Oxadiazoles Clubbed with Substituted Azetidin-2-one: A New Class of Potential Antimicrobial and Antitubercular. Drug Res (Stuttg) 2021, 71, 26-35, https://doi.org/10.1055/a-1252-2378. 
40. Rehman, A.; Akhtar, S.; Siddiqui, M.H.; Sayeed, U.; Ahmad, S.S.; Arif, J.M.; Khan, M.K.A. Identification of potential leads against 4-hydroxytetrahydrodipicolinate synthase from Mycobacterium tuberculosis. Bioinformation 2016, 12, 400-407, https://doi.org/10.6026/97320630012400.

41. Guedes, I.A.; Pereira, F.S.S.; Dardenne, L.E. Empirical Scoring Functions for Structure-Based Virtual Screening: Applications, Critical Aspects, and Challenges. Frontiers in Pharmacology 2018, 9, https://doi.org/10.3389/fphar.2018.01089.

42. Laxmi, B.; Sant Kumar, V.; Akhlesh Kumar, J.; Suresh, T. Lead Molecules as Novel Aromatase Inhibitors: In Silico De Novo Designing and Binding Affinity Studies. Letters in Drug Design \& Discovery 2020, 17, 655-665, https://doi.org/10.2174/1570180816666190703152659.

43. Nasab, R.; Hassanzadeh, F.; Khodarahmi, G.; Rostami, M.; Mirzaei, M.; Jahanian-Najafabadi, A.; Mansourian, M. Docking study, synthesis and antimicrobial evaluation of some novel 4-anilinoquinazoline derivatives. 2017, 12, 425-433, https://doi.org/10.4103/1735-5362.213988.

44. Morris, G.M.; Goodsell, D.S.; Huey, R.; Olson, A.J. Distributed automated docking of flexible ligands to proteins: Parallel applications of AutoDock 2.4. Journal of Computer-Aided Molecular Design 1996, 10, 293-304, https://doi.org/10.1007/BF00124499.

45. Khan, F.I.; Lai, D.; Anwer, R.; Azim, I.; Khan, M.K.A. Identifying novel sphingosine kinase 1 inhibitors as therapeutics against breast cancer. Journal of Enzyme Inhibition and Medicinal Chemistry 2020, 35, 172186, https://doi.org/10.1080/14756366.2019.1692828.

46. Khan, M.K.A.; Akhtar, S.; Arif, J.M. Structural Insight into the Mechanism of Dibenzo[a,1]pyrene and Benzo[a]pyrene-Mediated Cell Proliferation Using Molecular Docking Simulations. Interdisciplinary Sciences: Computational Life Sciences 2018, 10, 653-673, https://doi.org/10.1007/s12539-017-0226-7.

47. Khan, M.K.A.; Akhtar, S.; Arif, J.M. Development of In Silico Protocols to Predict Structural Insights into the Metabolic Activation Pathways of Xenobiotics. Interdisciplinary Sciences: Computational Life Sciences 2018, 10, 329-345, https://doi.org/10.1007/s12539-017-0237-4. 